\title{
The Universal Calibration of Polyaluminocarbosilane and Determination of Mark-Houwink Constant
}

\author{
ZHAO Dafang ${ }^{1, a^{*}}$, LI Xiaodong ${ }^{2, b}$ \\ ${ }^{1}$ AVIC Composites company LTD. , Beijing, 101416, China; \\ ${ }^{2}$ National University of Defense Technology, Changsha, 410073, China \\ adafang503@hotmail.com, bxdli0153@sina.com
}

Keywords: Polycarbosilane; Polyaluminocabosilane; Mark-Houwink constant Abstract. Polyaluminocarbosilane (PACS) is an important precursor of SiC ceramics. And the molecular distribution of PACS has an essential influence on the properties of its derived ceramics. The importance of absolute molecular weight analysis of PACS and the convenience of gel permeation chromatography ( GPC) make GPC using universal calibration curving an attractive way for PACS characterization. In this paper, the GPC calibration curve of PACS at $25{ }^{\circ} \mathrm{C}$ in tetrahydrofurran (THF), was determined by lsqnonlin non-linear function optimization in matlab, along with vapor pressure osmometry (VPO) and GPC characterization. With the calibration curve and GPC columns, the Mark-Houwink constant of PACS was obtained $\left(K_{\mathrm{PACS}}=1.93 \times 10^{-3} \mathrm{ml} / \mathrm{g}\right.$, $\alpha_{\text {PACS }}=0.75$ )

\section{Introduction}

Polycarbosilane (PCS) is a precursor for $\mathrm{SiC}$ ceramics with $-\mathrm{Si}-\mathrm{C}$ - main chains ${ }^{[1]}$. A series of $\mathrm{SiC}$ ceramics can be obtained from PCS, such as nano-powder, films, fibers and ceramic matrix composites, taking advantage of its good processing properties as polymers ${ }^{[2-5]}$. Polyaluminocarbosilane (PACS) is a precursor for $\mathrm{SiC}$ ceramics containing aluminum by introducing $\mathrm{Al}$ atom into PCS molecular structure ${ }^{[6,7]}$ ( the structure unit of PACS and PCS was shown in Fig.1). As an important precursor of ultra-high-temperature-resistant SiC fibers, PACS has a wide application potential in the fields of aerospace and nuclear energy ${ }^{[6,8]}$. The molecular distribution and absolute molecular weight analysis of PACS has an essential influence on properties of its derived ceramics. Therefore, the importance of absolute molecular weight analysis of PACS and the convenience of gel permeation chromatography ( GPC) make GPC using universal calibration curving an attractive way for PACS characterization. It is proved that the universal calibration was suitable for PCS in tetrahydrofuran $(\mathrm{THF})^{[9]}$. The calibration curve and the Mark-Houwink constant of PCS have been determined, along with multi-angle laser light scattering (MALLS) and GPC characterization ${ }^{[9]}$. The molecular structure of PCS and PACS is partly similar (as shown in Fig.1), as PACS becomes PCS when aluminum content is zero. In our work, the GPC calibration curve of PACS at $25^{\circ} \mathrm{C}$ in THF, can be determined by lsqnonlin non-linear function optimization in matlab, along with vapor pressure osmometry (VPO) and GPC characterization. With the calibration curve and GPC columns, the Mark-Houwink constant of PACS was obtained.

\section{Experimental}

PACS and PCS was synthesized following the references ${ }^{[10]}$. The number average molecular weight $(\overline{M n})$ of PACS and PCS was measured by K-7000 vapor pressure osmometer. Molecular weight distributions were measured by GPC (gel permeation chromatography, Waters-1515) with HR0.5 and HR3 s-Styragel columns calibrated with polystyrene standards and with THF as eluent as a flow rate of $0.5 \mathrm{ml} / \mathrm{min}$. 


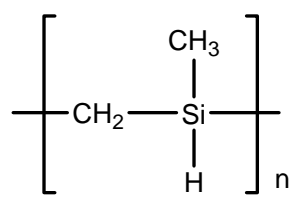

(a) repeat unit of PCS

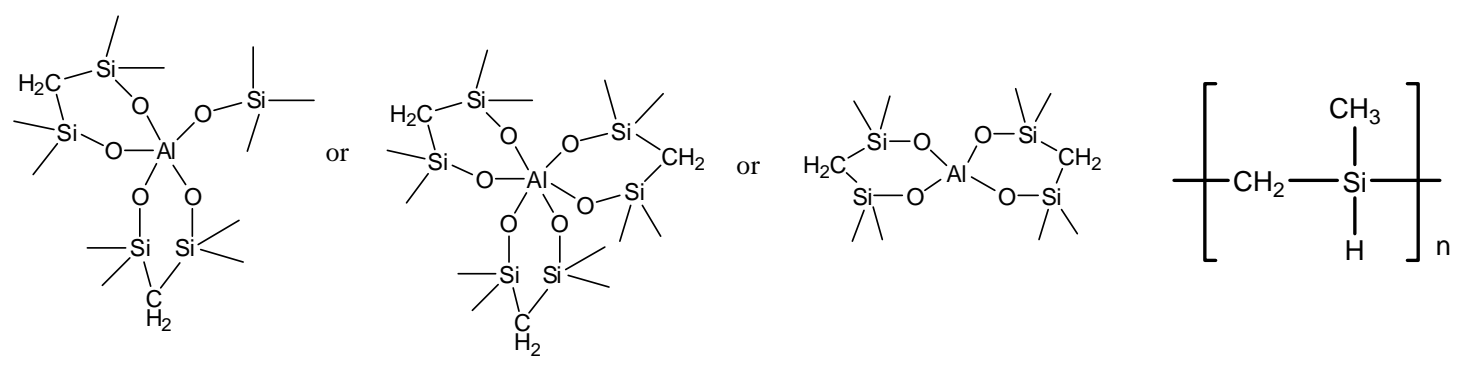

(b) typical structure of PACS

Fig. 1 Typical structure of PCS and PACS

\section{Results and discussions}

Universal calibration of PACS. 3 samples of PACS and 3 samples of PCS with increasing molecular weight were selected for determination of GPC calibration curve. The obtained GPC curves of PACS and PCS samples were shown in Fig.2. And the number average molecular weight $\mathrm{M}_{\mathrm{VPO}}$ of samples measured by VPO method were listed in Table 1.
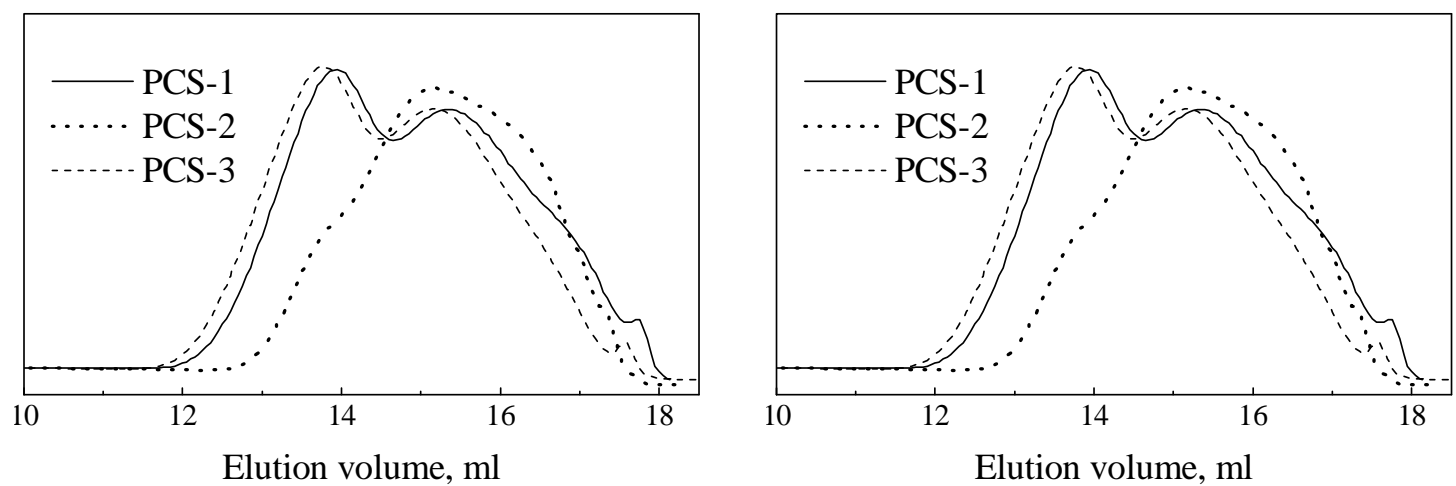

Fig.2 GPC curves of PCS and PACS for calibration curve determination

For a given chromatographic column used in GPC measurements, the relationship of molecular weight $M$ and elution volume $V e$ is usually described as follow:

$$
\log M=A-B V e
$$

Whereas $A$ and $B$ is parameters which is related with solute, solvent, temperature, carrier and instrument structure.

The $\overline{M n}$ was calculated from GPC results based on spline method as follow:

$$
\overline{M n}=\frac{\sum_{i} H_{i}}{\sum_{i} \frac{H_{i}}{M_{i}}}=\frac{\sum_{i} H_{i}}{\sum_{i} \frac{H_{i}}{\exp \left(A-B V e_{i}\right)}}
$$

20 sampling points were taken per $\mathrm{ml}$, and the number of equant chips was determined by the width of GPC curves. The range of integration and the corresponding number of chips was list in Table 1 . The $\overline{M n}$ was calculated with the obtained data, which should be equal to $M_{V P O}$ : 


$$
\overline{M n}=M_{V P O}
$$

The value of $A$ and $B$ can be solved with equation (2) and (3).

The error is defined as $e=M_{V P O}-\overline{M n}$. The values of $A$ and $B$ obtained should keep each group of error sum squares $\sum_{i=1}^{6} e_{i}^{2}$ minimal. According to the above-mentioned principle, the value of $A$ and $B$ was solved by lsqnonlin non-linear function optimization in matlab. The obtained PACS and PCS calibration curve is listed as follow:

$$
\log M=9.17-0.389 \mathrm{Ve}
$$

Putting the value of $\mathrm{A}$ and $\mathrm{B}$ into Eq. (2), each series of $\overline{M n}_{i}$ was calculated, and listed in Table 1.

Table 1 Integration range, chip number, measured and calculated $\overline{M n}$ of PACS and PCS

\begin{tabular}{lcccc}
\hline & & & \multicolumn{2}{c}{$\overline{M n}$} \\
\cline { 4 - 5 } Samples & Integration range $[\mathrm{ml}]$ & Chip number & $\mathrm{M}_{\mathrm{VPO}}$ & Calculated \\
\hline PACS-1 & $9.8 \sim 18.55$ & 176 & 1093 & 943 \\
PACS-2 & $7.75 \sim 19.15$ & 229 & 2005 & 1951 \\
PACS-3 & $7.5 \sim 18.1$ & 212 & 2282 & 2196 \\
PCS-1 & $11.9 \sim 18.1$ & 124 & 1353 & 1257 \\
PCS-2 & $12.5 \sim 18$ & 110 & 840 & 1038 \\
PCS-3 & $11.6 \sim 18.1$ & 130 & 1772 & 1691 \\
\hline
\end{tabular}

From Table 1, it is found that the calculated $\overline{M n}$ values approximately agree with the $M_{\mathrm{VPO}}$ measured by VPO. Therefore, the GPC curve of PACS can be calibrated with Eq.(4).

Determination of Mark-Houwink constant. Suppose the universal calibration was suitable for both PACS, PCS and polystyrene (PS), then, for a given elution volume $V e$, the ${ }^{[\eta] M}$ of PS, PCS and PACS is constant. It is concluded that the Eq.(5) is workable:

$$
[\eta]_{P A C S} M_{P A C S}=[\eta]_{P S} M_{P S}
$$

According to Mark-Houwink equation,

$$
[\eta]_{P A C S}=K_{P A C S} M_{P A C S}^{\alpha_{P A C S}}
$$

And

$$
[\eta]_{P S}=K_{P S} M_{P S}^{\alpha_{P S}}
$$

Putting Eq.(6) and Eq.(7) into Eq.(5), it is obtained as follow : 


$$
\log K_{P S} M_{P S}^{\alpha_{P S}+1}=\log K_{P A C S} M_{P A C S}^{\alpha_{P A C S}+1}
$$

The value of $K$ and $\alpha$ is list as follow: $\mathrm{K}_{\mathrm{PS}}=0.011 \mathrm{ml} / \mathrm{g}, \alpha_{\mathrm{PS}}=0.725^{[11]}$. Putting them into Eq.(8), it is obtained that :

$$
\log K_{P A C S}+\left(\alpha_{P A C S}+1\right) M_{P A C S}=-1.959+1.725 \log M_{P S}
$$

Putting the calibration curves into Eq. (9), it is obtained that:

$$
\log K_{P A C S} M_{P A C S}^{\alpha_{P A C S}+1}=13.34-0.681 V e
$$

The values of $K$ and $\alpha$ of PACS were solved with Eq.(10) and Eq.(4): $K_{\text {PACS }}=1.93 \times 10^{-3} \mathrm{ml} / \mathrm{g}$, $\alpha_{\text {PACS }}=0.75$.

\section{Summary}

In the work, a method to universal calibration of PACS without solution viscosity measurement and precipitation fractionation was offered. The GPC calibration curve of PACS at $25 \square$ in the THF, was determined by lsqnonlin non-linear function optimization in matlab, along with VPO and GPC characterization. With the calibration curve and GPC columns, the Mark-Houwink constant of PACS can be obtained

\section{References}

[1] S. Yajima, Y. Hasegawa, J. Hayashi, Synthesis of continuous silicon carbide fibre with high tensile strength and high Young's modulus, J. Mater. Sci., 13 (1978) 2569-2576.

[2] R.A. Wach, M. Sugimoto, A. Idesaki, Molecular sieve SiC-based membrane for hydrogen separation produced by radiation curing of preceramic polymers, Materials Science and Engineering: B, 140 (2007) 81-89.

[3] H. M. Yen, S. Jou,C. J. Chu,Si-O-C nanotubes from pyrolyzing polycarbosilane in a mesoporous template, Materials Science and Engineering: B, 122 (2005) 240-245.

[4] J. S. Lee,T. Yano, Fabrication of short-fiber-reinforced SiC composites by polycarbosilane infiltration, J. Eur. Ceram. Soc., 24 (2004) 25-31.

[5] T. Shimoo, K. Okamura,H. Takeuchi, Effect of reduced pressure on oxidation and thermal stability of polycarbosilane-derived SiC fibers, J. Mater. Sci., 38 (2003) 4973-4979.

[6] T. Ishikawa, Y. Kohtoku, K. Kumagawa, High-strength alkali-resistant sintered SiC fibre stable to $2,200^{\circ} \mathrm{C}$, Nature, 391 (1998) 773-775.

[7] F. Cao, D.P. Kim, X.D. Li, Synthesis of polyaluminocarbosilane and reaction mechanism study, J. Appl. Polym. Sci., 85 (2002) 2787-2792.

[8] D. F. Zhao, X. D. Li, H. Wang, Effects of oxygen content on the properties of Ultra-High-Temperature resistant Si-Al-C fibers, Key Engineering Materials, 368-372 (2008) 1774-1777.

[9] R. A. He, W. N. Hu, Universal calibration of polycarbosilane and determination of Mark-Houwink constant, Polym. Mater. Sci. Eng., (2008) 111-114.

[10]D. F. Zhao, X. D. Li, C. M. Zheng, Production mechanism of polyaluminocarbosilane using aluminum acetylacetonate with polysilacarbosilane, Journal of University of Science and Technology Beijing, (2007) 130-134

[11]W. Liu, B. G. Li, J. Shen, Determination and application of Mark-Houwink constant of syndiotactic polystyrene. Polym. Mater. Sci. Eng., 19 (2003) 187-189 